\title{
REDUCTION OF VOLATILE ORGANIC COMPOUNDS USING PHOTOCATALYSTS ACTIVATED BY BLUE LED
}

\author{
Atsuko Oyama \\ Material Technology Division,Tokushima Prefectural Industrial Technology Center, 11-2 nishibari saikacho \\ Tokushimacity,7708021,Japan \\ oyama@itc.pref.tokushima.jp
}

Kei-ichiro Murai

Faculty of Engineering, The university of Tokushima.2-1 minamijyosanjima, Tokushimacity, 7708506, Japan murai@chem.tokushima-u.ac.jp

Toshihiro Moriga

Faculty of Engineering, The university of Tokushima.2-1 minamijyosanjima, Tokushimacity, 7708506, Japan

moriga@chem.tokushima-u.ac.jp

\begin{abstract}
The effective utilisation of solar radiation requires the development of novel photocatalysts activated by visible-light. Activity tests of toluene decomposition were carried out in the presence of modified titanium dioxide photocatalysts in which some oxygen ions were substituted by sulfur. The elimination of toluene after 1 hour irradiation of the photocatalysts by a pulsed blue LED yielded 44 percent elimination. Pretreatment in which $\mathrm{S}$-doped $\mathrm{TiO}_{2}$ was irradiated by $\mathrm{UV}$ for 1 hour prior to the activity test was effective in decomposing acetaldehyde, even under a pulsed green LED irradiation.
\end{abstract}

Keywords: Photocatalysts; VOCs; LEDs; visible-lights.

\section{Introduction}

Photocatalysts decompose some organic compounds on a surface when the catalyst is irradiated with UV light. Research by the Tokushima Prefectural Industrial Technology Center found that the photocatalytic decomposition of acetaldehyde by titanium dioxide under pulsed UV-LED irradiation (50\% duty ratio with pulse period of $1 \mathrm{~ms}$ ) showed the same yield as that under continuous UV-LED irradiation, and was especially effective in the decomposition of acetaldehyde at low concentration[1,2]. UV-LEDs are currently expensive but may enable the construction of some compact, high efficiency devices. Recently, much attention has been paid to visible-light-driven photocatalysts from the viewpoint of effective use of sunlight. In this study, oxygen in titanium dioxide photocatalyst was partly substituted by sulfur to improve activation by visible-light, and 
activity tests of decomposition of toluene and acetaldehyde, one of the volatile organic compounds (VOCs), were performed using pulsed or continuous blue and green LED irradiations.

\section{Experimental}

Commercially available titanium dioxide ST-01(Ishihara Sangyo) and the thiourea (typically $\mathrm{TiO}_{2}$ : thiourea $=1: 1$ in molar ratio) were mixed[3], and calcined at $400^{\circ} \mathrm{C}$ for 3 hours so that some oxygens were substituted by sulfurs and the resultant $\mathrm{S}-\mathrm{TiO}_{2}$ is subject to testing of activity with visible light. After the catalyst was mixed with water in a Petri dish to make a slurry, it was dried at $60{ }^{\circ} \mathrm{C}$. The dish containing the powder was put in a sampling bag, the inside of which was filled with toluene or acetaldehyde. The bag was left in a dark room for half a day to reach adsorption equilibrium. The continuous or pulsed irradiation by blue and green LEDs (manufactured by Nichia Corporation) was applied from the upper part of the bag (Fig.1), controlling the pulse width and the duty cycle (lighting time/ pulse period). The time evolution of the gas concentration in the bag was calculated from the initial and final concentrations. For reference, S-doped $\mathrm{TiO}_{2}$ irradiated by UV for 1 hour prior to the test was also prepared, to decompose previouslyadsorbed VOCs on the $\mathrm{TiO}_{2}$ surface [4].

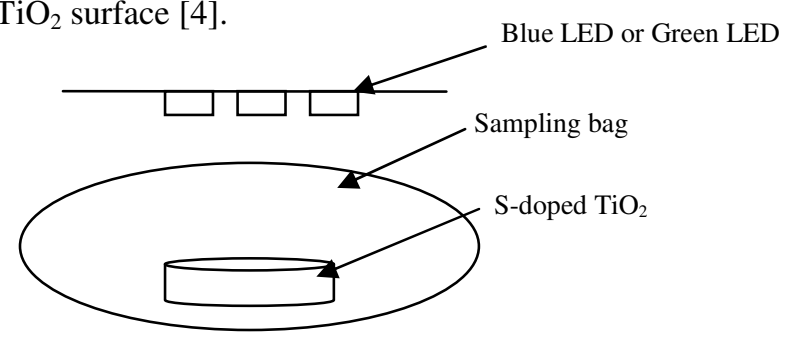

Fig.1 Light LED visible and experiment device chart of photocatalyst.

\section{Results and discussions}

\subsection{Photocatalytic activity against toluene}

Applying continuous irradiation by using a green LED (wavelength 520nm), or a blue

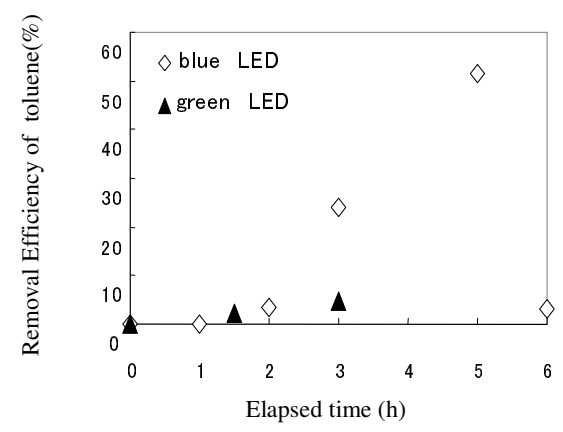

Fig.2. Difference of removal efficiency of toluene using green and blue LEDs, respectively. 
LED (wavelength 470nm), to the sample, was carried out to assess activity against $100 \mathrm{ppm}$ of toluene as the initial concentration. For the green LED, the toluene was only slightly removed, regardless of irradiation time. In contrast, for the blue LED, decomposition efficiency for toluene reached $50 \%$ after five hours, but decreased rapidly with elapsed time (Fig.2).

To confirm effectiveness of the pulsed irradiation for the photocatalytic decomposition, the pulsed blue LED irradiation (50\% duty cycle and 10001x in illuminance with pulse period $1 \mathrm{~ms}$ ) was applied to $7 \mathrm{ppm}$ of toluene as the initial concentration. The elimination ratio after 1 hour reached $44 \%$ at maximum (Fig.3).

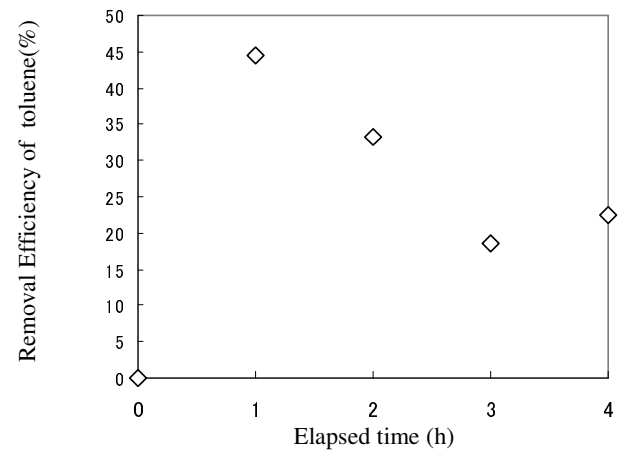

Fig.3. Removal efficiency of toluene when duty ratio applied was $50 \%$.

Figure 4 shows the removal efficiency of $11 \mathrm{ppm}$ of toluene under similarly controlled blue LED pulse irradiation (except that a $20 \%$ duty cycle applied). The low concentration toluene could be removed under the pulsed irradiation with small power consumption. Though toluene is reported to be hard to decompose [5], the visible-light-driven titanium dioxides were activated under an illuminance of as low as 10001x of the blue LED. As the illuminance at workspaces in offices is around 1000lx, this result suggests the possibility of photocatalytic decomposition of VOCs using $\mathrm{TiO}_{2}$ under domestic lighting.

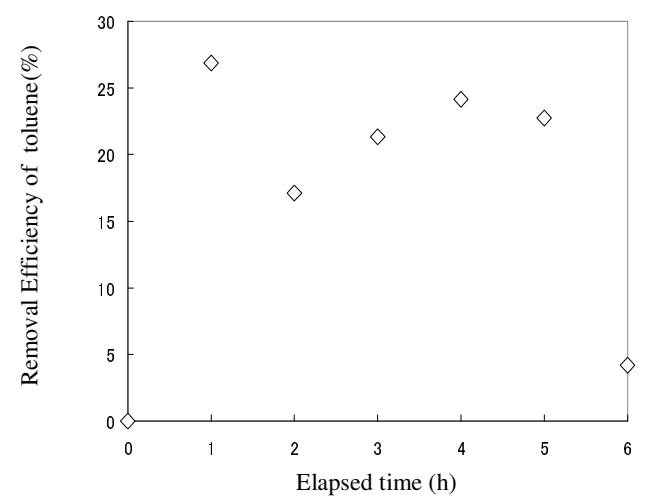

Fig.4. Removal efficiency of toluene when duty ratio applied was $20 \%$. 


\subsection{The schematic model of decomposition and adsorption when continuous and pulsed LED irradiation.}

Continuous LED irradiation

\begin{tabular}{|l|l|l|l|l|}
\hline ON & ON & ON & ON & ON \\
\hline
\end{tabular}

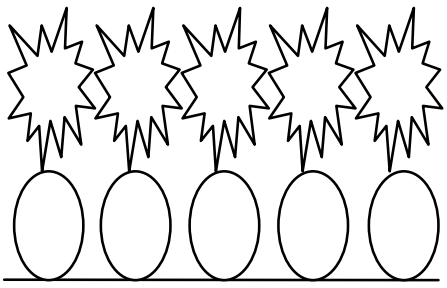

Surface of S-doped $\mathrm{TiO}_{2}$
Pulsed LED irradiation

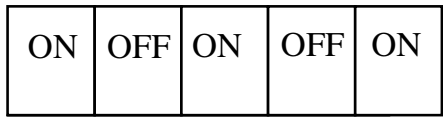

adsorption

decomposition

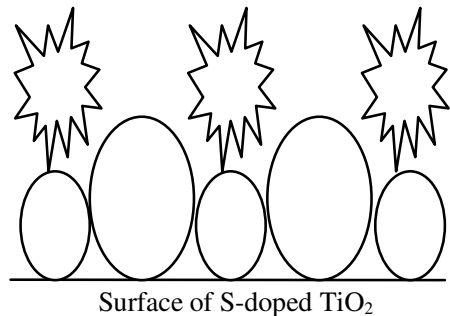

Fig.5. Proposed scheme of decomposition and adsorption mechanisms of VOCs under a continuous (left) and a pulsed (right) LED irradiations.

In addition, we have proposed decomposition and adsorption mechanisms of VOCs in the presence of continuous and pulsed LED irradiations (Fig.5) [1,2]. During the continuous LED irradiation, adsorption and decomposition of VOCs occurs at the same time. In contrast, when the LEDs are turned off, only adsorption behavior occurs and decomposition no longer takes place. Generation of electron-hole pairs at the catalyst surface no longer occurs in the absence of the irradiation and residual active species such as $\mathrm{O}_{2} \cdot{ }^{-}$and $\mathrm{OH} \cdot$ radicals, which form through reactions between an $\mathrm{O}_{2}$ molecule and an electron and between a $\mathrm{H}_{2} \mathrm{O}$ molecule and a hole, respectively, cause the decomposition of VOCs [6]. The decomposition rate in the absence of the irradiation was thus much lower than that in the presence of irradiation. Thus we propose that the S-doped $\mathrm{TiO}_{2}$ will have enough time to desorb the decomposition products and to absorb more VOCs in preparation for the next LED irradiations while the LED is turned off.

Wang et al reported [7] photocatalytic decomposition of Reactive Red 22 in aqueous suspension of $\mathrm{TiO}_{2}$ under a pulsed UV LED irradiation with the pulse width of $0.1 \mathrm{~s}$ in the duty cycle of $9.1 \%(=0.1 \mathrm{~s} / 1.1 \mathrm{~s})$. The apparent rate constants for experiments conducted in pulsed, irradiation were 1.5-2 times lower than for those experiments conducted under continuous irradiation. However, the photons emitted from the LED operated under the pulsed irradiation were 10 times lower than those under continuous irradiation Therefore, the overall photo efficiency for experiments conducted with the pulsed irradiation was much higher than those for experiments with continuous irradiation.

As a consequence, the pulsed irradiation has a comparable in efficiency for removal of VOCs to the continuous irradiation. If the period for irradiation is appropriate against the 
timescale for absorption, decomposition and removal behavior, the efficiency will be much improved.

\subsection{Photocatalytic Activity against Acetaldehyde}

Acetaldehyde is reported to be more easily decomposed than toluene. Fig. 6 shows the results of using blue LED continuous irradiation (illuminance: 1000lx, acetaldehyde of initial concentration: 50ppm). Concentration of acetaldehyde decreased with elapsed time, and five hours later its yield reached $46 \%$ at maximum.

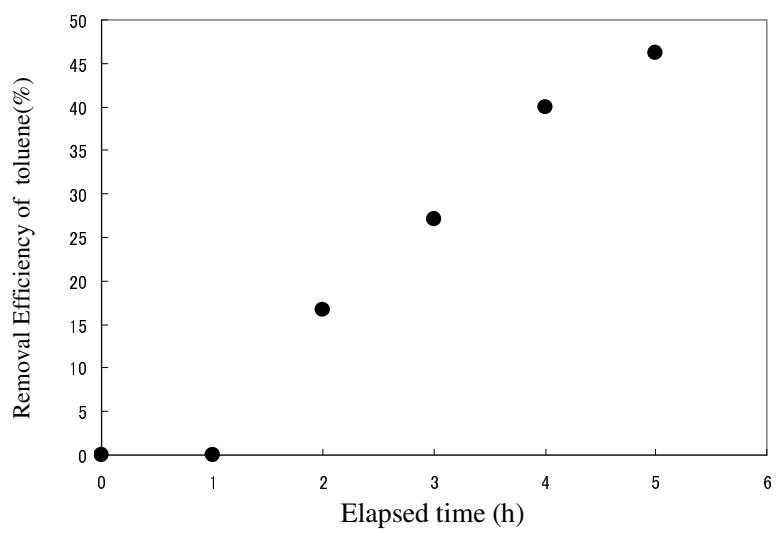

Fig.6. Removal efficiency of acetaldehyde under blue LED irradiation.

The effect of the pretreatment of the S-doped $\mathrm{TiO}_{2}$ onphotocatalytic decomposition of acetaldehyde with as high an initial concentration as $500 \mathrm{ppm}$ was examined under continuous green LED irradiation. The elimination ratio without the exposure to ultraviolet radiation as the pretreatment was less than $10 \%$. However, the preprocessed sample reached an elimination ratio of $35 \%$ after one hour, and showed a five times higher elimination ratio (Fig.7).

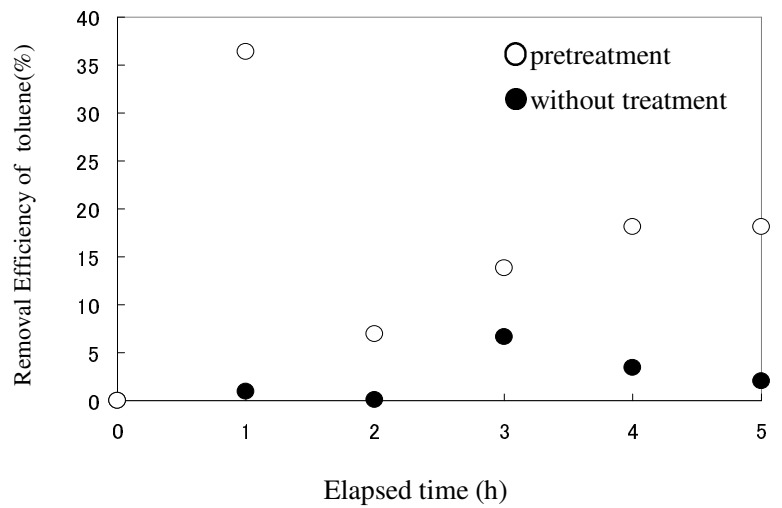

Fig.7. Removal efficiency of acetaldehyde by preprocessing photocatalysts. 
Impurities on the catalytic surface and the adsorbing species would be removed by preprocessing, that is, the exposure to ultraviolet radiation, and as a result, it is thought that even the green LED irradiation may be able to decompose acetaldehyde adsorbed on the cleaned surface. If the surface was not cleaned by pretreatment, the number of the activesites for adsorbing such VOCs would decrease. When visible LEDs were used as a light source for photocatalysts, such a pretreatment as UV irradiation of the S-doped $\mathrm{TiO}_{2}$ will improve the efficiency and the decomposition ratio.

\section{Conclusions}

Pulsed irradiation by visible-light LEDs was as effective in the photocatalytic decomposition of VOCs by S-doped $\mathrm{TiO}_{2}$ as that of the UV-LEDs previously reported to be effective. Because the pulsed irradiation will lead to a low electric power consumption compared with continuous irradiation, further conservation of energy can be expected.

\section{References}

1. Y. Hirashima, T.Kagawa and T. Matsubara, Report of Tokushima Prefectural Industrial Technology Center,Vol.14, 2005, p.34-36,

2. Y. Hirashima and T. Kagawa, Report of Tokushima Prefectural Industrial Technology Center,Vol.16, 2007, p.1-5

3. T.Ohno, Photocatalysis, Vol. 19, 2006, p.91-102.

4. T.Sano, Chantal Guillard, Christaphe Geantet, N.Negishi, Photocatalysis Vol. 27, 2008, p.84-85.

5. R.Abe et al, Photocatalysis Vol. 33, 2010, p.66-67.

6. K.Oda et al, Photocatalysis Vol.27, 2008, p.102-103.

7. Wen-Yu Wang et al, Water Research 40, 2006, 2249-2258. 